TOCSY Spectra. 2D-TOCSY spectra were used to identify spin systems corresponding to individual amino acids in $\mathbf{1 - 3}$ (Figs S1-S3). The presence of essentially one peptidic species (2) in $\mathrm{H}_{2} \mathrm{O}: \mathrm{D}_{2} \mathrm{O}$ was suggested in the initial product spectrum (Fig. S2) as were two peptides (2+3) at equilibrium (Fig. S3), since 8 and 16 spin systems were respectively present. Chemical shift assignments for $\mathbf{1 - 3}$ are summarized in Table S1 and clearly distinguish between these species. 


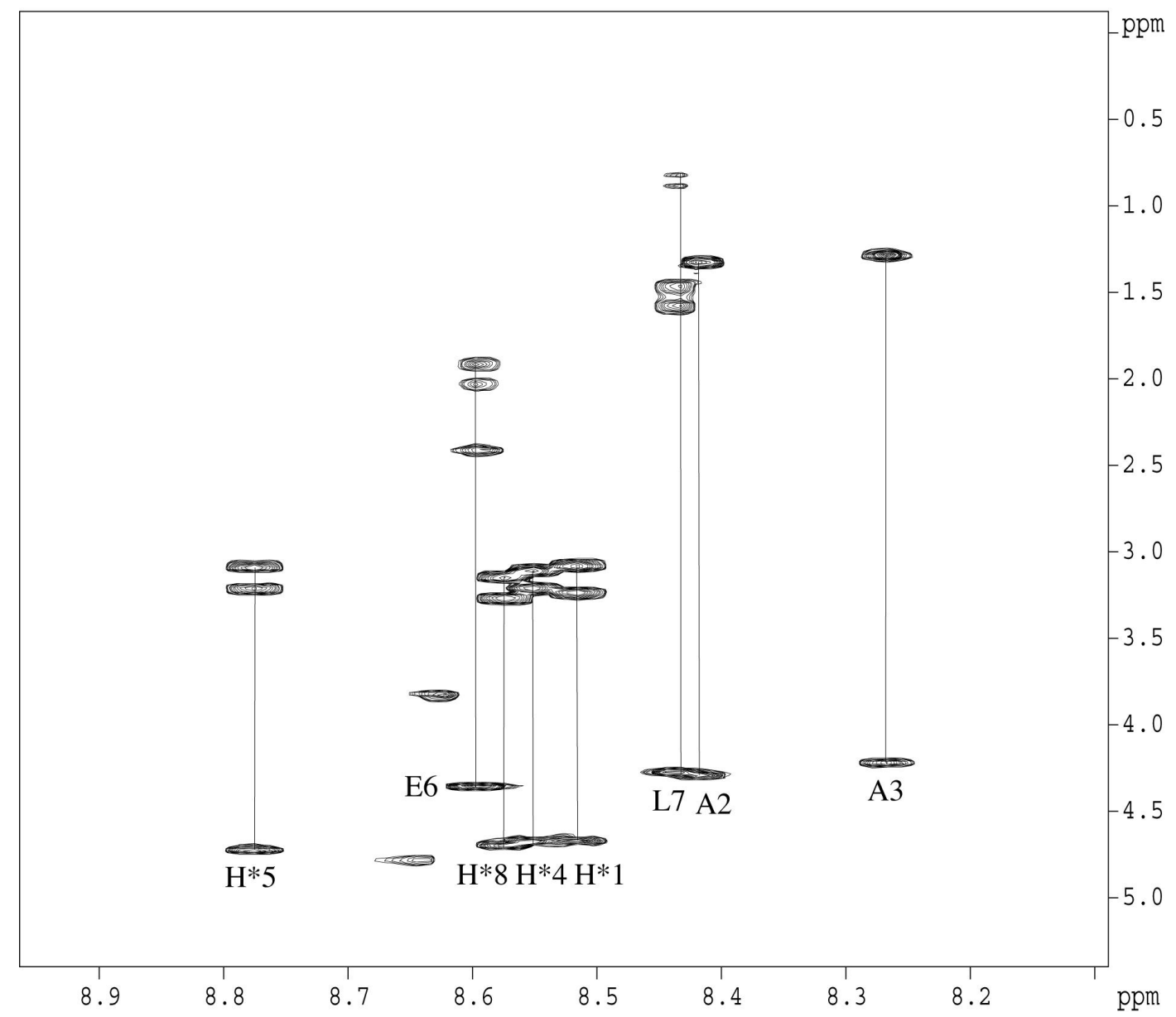

Figure S1. Fingerprint region of the $600 \mathrm{MHz}$ TOCSY spectrum (298 K) for Ac$\mathrm{H}^{*} \mathrm{AAH} * \mathrm{H}^{*} \mathrm{ELH}^{*}-\mathrm{NH}_{2}$ (1) in $\mathrm{H}_{2} 0: \mathrm{D}_{2} 0$ (9:1). Amino acid spin-systems are identified by standard one-letter codes, their sequence position by solid lines connecting the cross-peaks. 


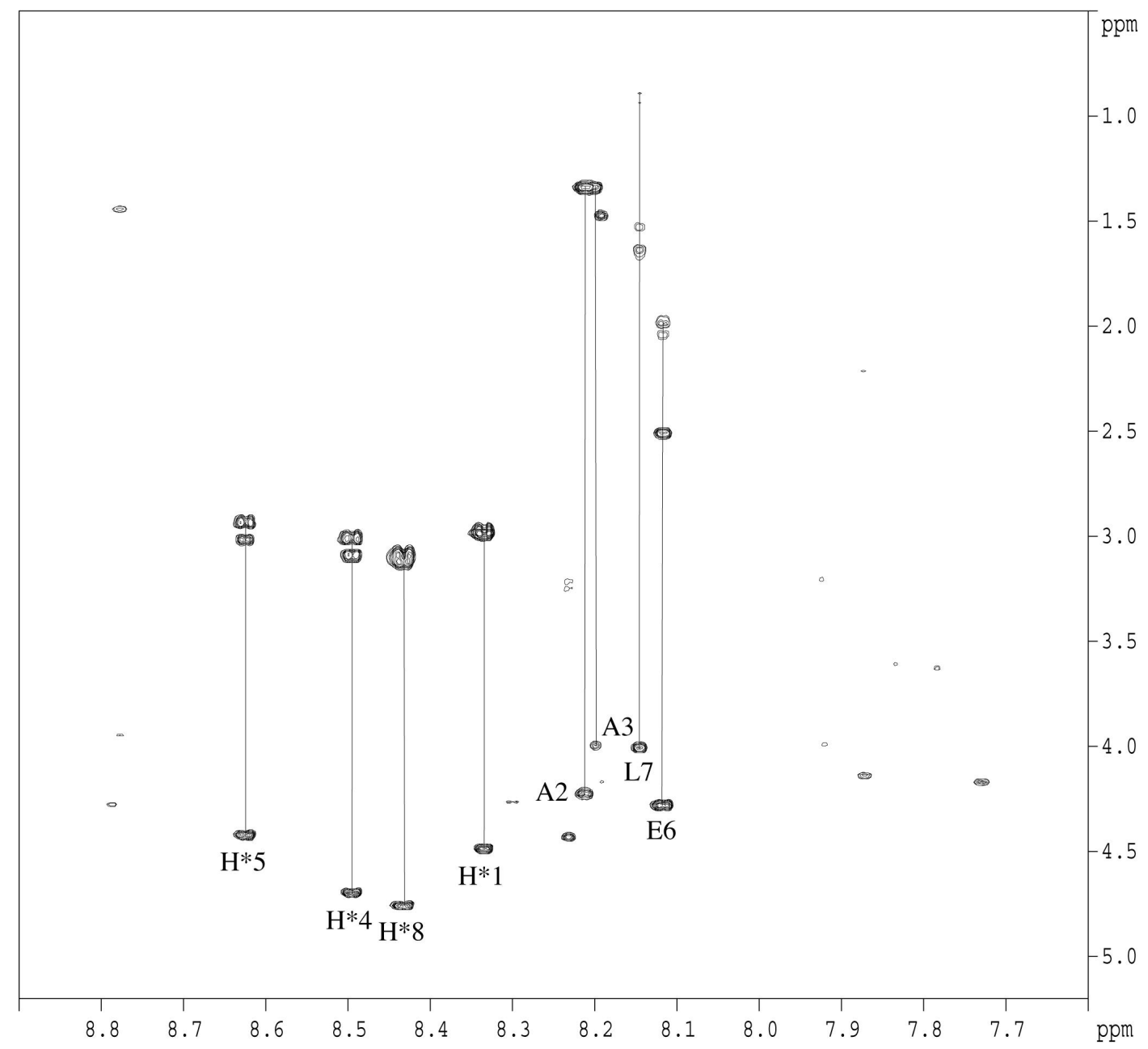

Figure S2. Fingerprint region of the $600 \mathrm{MHz}$ TOCSY spectrum (298 K) for Ac$\mathrm{H}^{*} \mathrm{AAH} \mathrm{H}^{*} \mathrm{ELH}^{*}-\mathrm{NH}_{2}$ (1) in $\mathrm{H}_{2} 0: \mathrm{D}_{2} \mathrm{O}$ (9:1) after reaction with 2equivalents of $\left[\mathrm{Pd}\left({ }^{15} \mathrm{NH}_{2}\left(\mathrm{CH}_{2}\right)_{2}{ }^{15} \mathrm{NH}_{2}\right)\left(\mathrm{ONO}_{2}\right)_{2}\right]$. Spectrum recorded within 4 hours. Amino acid spin-systems corresponding to the major product $\mathbf{2}$ are identified by standard oneletter codes, their sequence position in $\mathbf{2}$ (in bold) and by solid lines connecting the cross-peaks. 


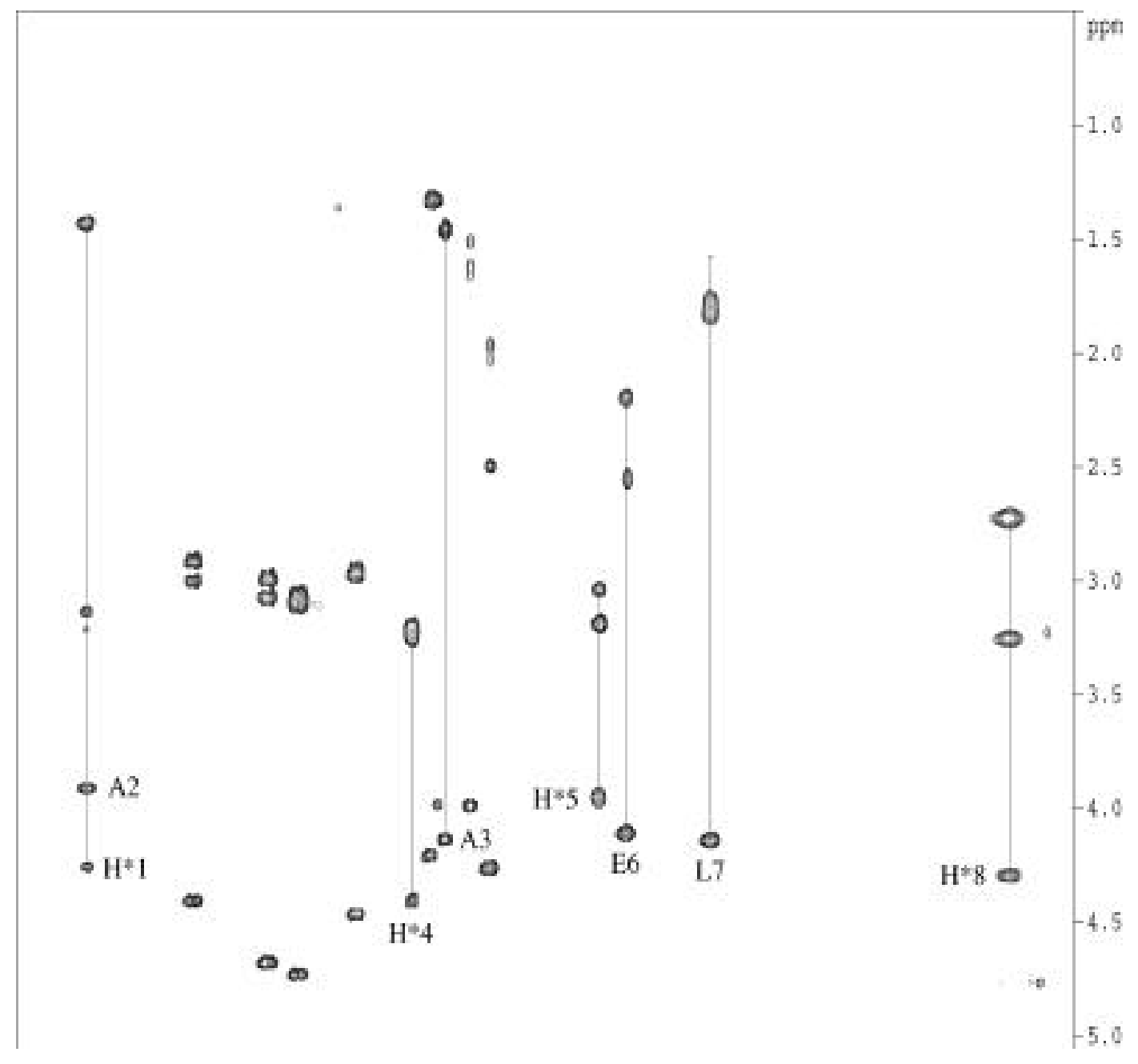

Figure S3. Fingerprint region of the $600 \mathrm{MHz}$ TOCSY spectrum $(298 \mathrm{~K})$ from the reaction of $\left[\mathrm{Pd}\left({ }^{15} \mathrm{NH}_{2}\left(\mathrm{CH}_{2}\right)_{2}{ }^{15} \mathrm{NH}_{2}\right)\left(\mathrm{ONO}_{2}\right)_{2}\right]$ and $\mathrm{Ac}-\mathrm{H}^{*} \mathrm{AAH} \mathrm{H}^{*} \mathrm{ELH}^{*}-\mathrm{NH}_{2}(\mathbf{1})$ in $\mathrm{H}_{2} 0: \mathrm{D}_{2} 0(9: 1)$ aged for 2 days. Amino acid spin-systems corresponding to the major product 3 are identified by standard one-letter codes, their sequence position in $\mathbf{3}$ (in bold) and by solid lines connecting the cross-peaks. 


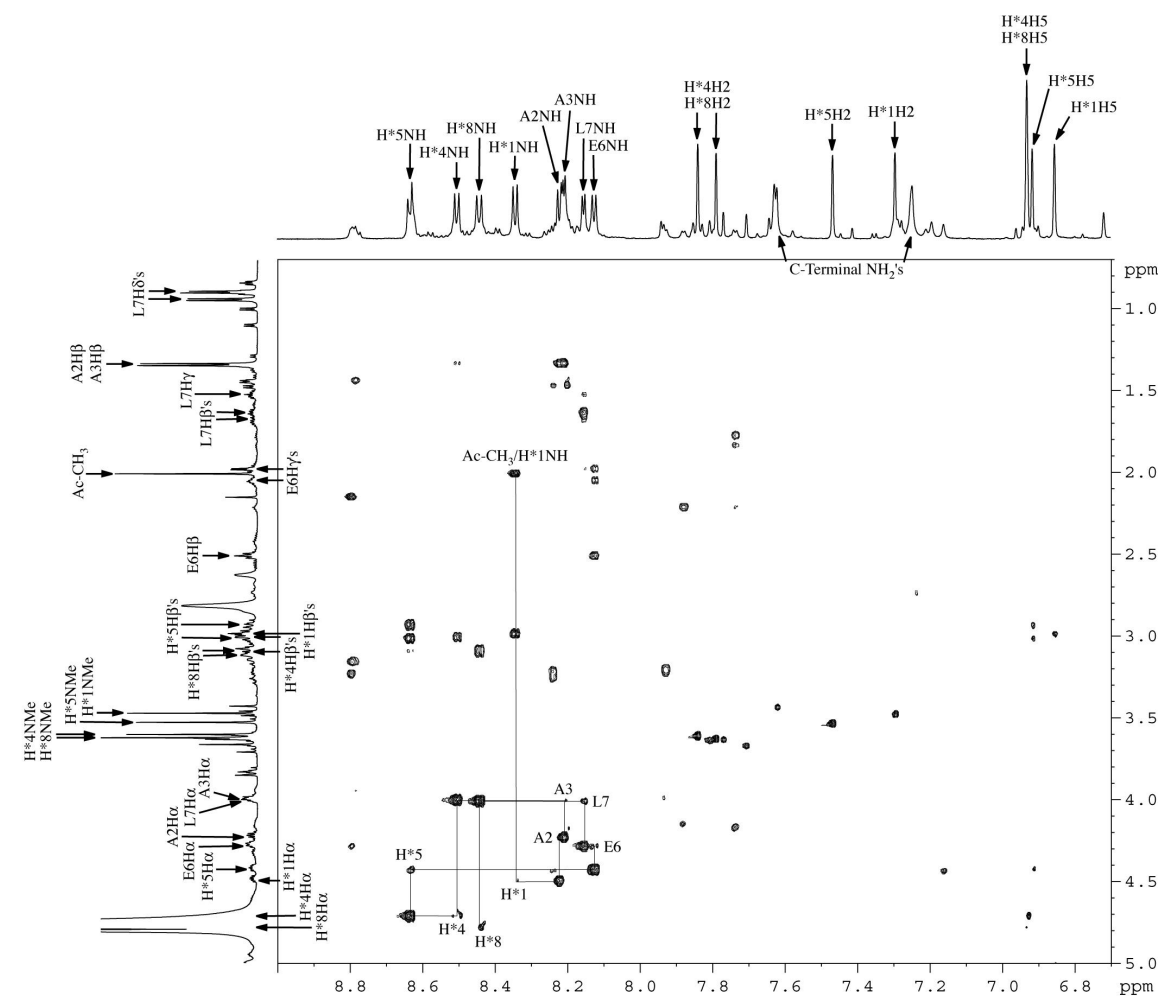

Figure S4. A section of the 600MHz NOESY spectrum of $\mathbf{2}$ (see conditions in Fig. S2 and text). Sequential connectivity cross peaks are labeled by standard one-letter codes andby residue numbers in $\mathbf{2}$. 


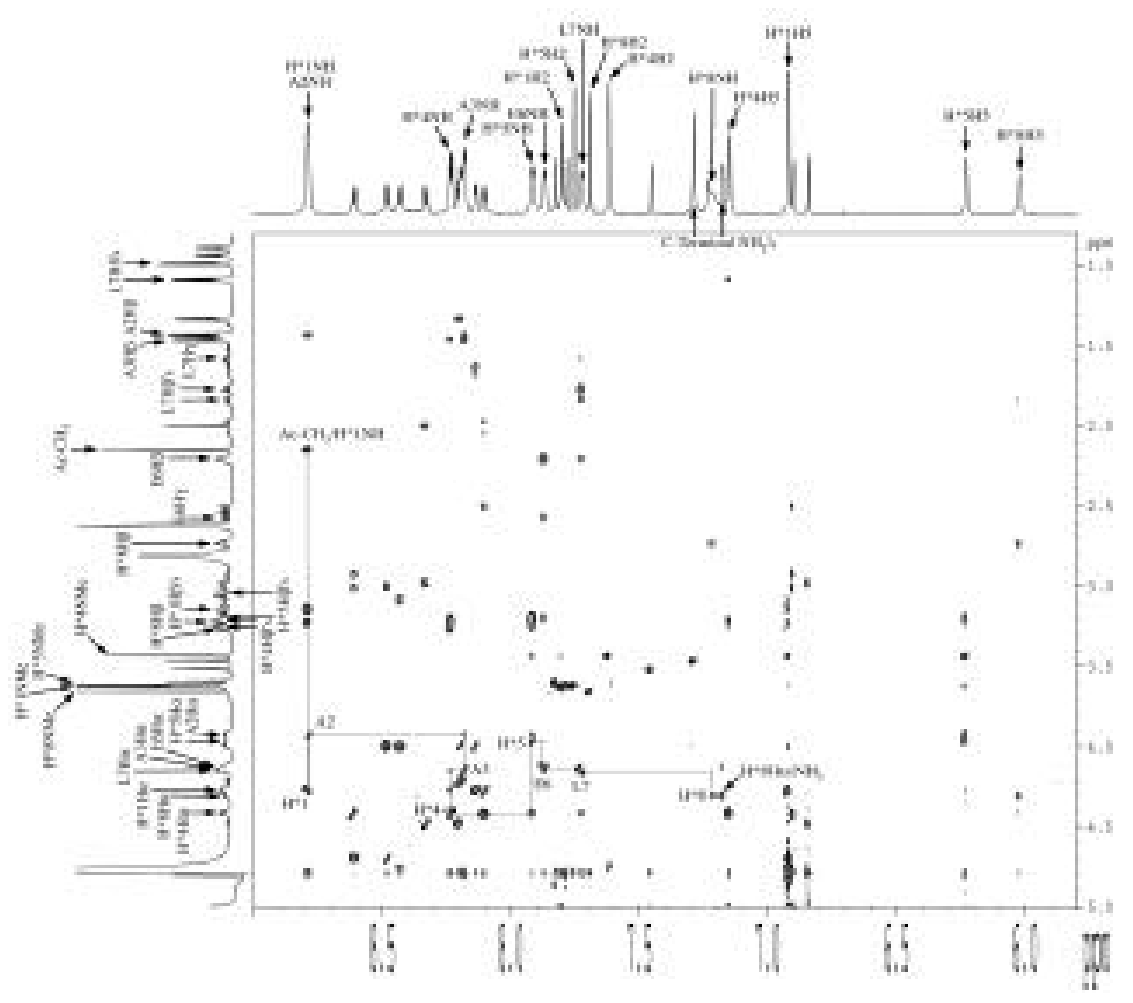

Figure S5. A section of the 600MHz ROESY spectrum of $\mathbf{3}$ (see conditions in Fig. S3 and text). Sequential connectivity cross peaks are labeled by standard one-letter codes andby residue numbers in $\mathbf{3}$. 
Table S1 : ${ }^{1} \mathrm{H}$ NMR resonance assignments and chemical shifts $(\delta \mathrm{ppm})$ for $\mathbf{1 - 3}$ in $\mathrm{H}_{2} \mathrm{O} / \mathrm{D}_{2} \mathrm{O}(9: 1)$ at $298 \mathrm{~K}$.

\begin{tabular}{|c|c|c|c|c|}
\hline Residue & NH & $\mathbf{H}_{\alpha}$ & $\mathbf{H}_{\beta}$ & Other \\
\hline \multicolumn{5}{|l|}{ Peptide 1} \\
\hline aAc-His*1 & 8.52 & 4.67 & $3.09,3.24$ & $\begin{array}{l}\mathrm{H} 28.62 ; \mathrm{H} 5 \text { 7.27; NMe 3.82- } \\
3.85 ;{ }^{\mathrm{a}} \mathrm{CH}_{3} 1.98\end{array}$ \\
\hline Ala2 & 8.42 & 4.29 & 1.33 & \\
\hline Ala3 & 8.27 & 4.22 & 1.29 & \\
\hline His*4 & 8.55 & 4.67 & $3.12,3.21$ & H2 8.62; H5 7.25; NMe 3.82-3.85 \\
\hline His*5 & 8.78 & 4.73 & $3.09,3.21$ & H2 8.62; H5 7.24; NMe 3.82-3.85 \\
\hline Glu6 & 8.60 & 4.35 & $1.92,2.03$ & $\mathrm{\gamma CH}_{2} 2.41$ \\
\hline Leu7 & 8.44 & 4.27 & 1.58 & $\gamma \mathrm{CH} 1.47 ; \delta \mathrm{CH}_{3} 0.83,0.89$ \\
\hline His*8- $\mathrm{NH}_{2}$ & 8.58 & 4.69 & $3.15,3.27$ & $\begin{array}{l}\mathrm{H} 28.62 ; \mathrm{H} 5 \text { 7.29; NMe 3.82- } \\
3.85 ; \mathrm{NH}_{2} 7.62,7.21\end{array}$ \\
\hline
\end{tabular}

\begin{tabular}{ccccl}
\hline Peptide 2 & & & & \\
\hline${ }^{\mathrm{a}}$ Ac-His*1 & 8.34 & 4.48 & 2.98 & $\begin{array}{l}\mathrm{H} 27.30 ; \mathrm{H} 56.86 ; \mathrm{NMe} 3.47 ; \\
{ }^{\mathrm{a}} \mathrm{CH}_{3} 2.01\end{array}$ \\
Ala2 & 8.22 & 4.22 & 1.34 & \\
Ala3 & 8.21 & 3.99 & 1.34 & \\
His*4 & 8.50 & 4.69 & $3.00,3.08$ & $\mathrm{H} 27.79$ or $7.84 ; \mathrm{H} 56.93 ; \mathrm{NMe}$ \\
& & & & 3.60 or 3.62 \\
His*5 & 8.63 & 4.41 & $2.93,3.01$ & $\mathrm{H} 27.47 ; \mathrm{H} 56.92 ; \mathrm{NMe} 3.53$ \\
Glu6 & 8.13 & 4.27 & 2.50 & $\gamma \mathrm{CH} 1.98,2.03$ \\
Leu7 & 8.15 & 4.00 & $1.63,1.67$ & $\gamma \mathrm{CH} 1.53 ; \delta \mathrm{CH}_{3} 0.89,0.94$ \\
His*8-NH & 8.44 & 4.75 & $3.09,3.11$ & $\mathrm{H} 27.79$ or $7.84 ; \mathrm{H} 56.93 ; \mathrm{NMe}$ \\
& & & & 3.60 or $3.62 ; \mathrm{NH}_{2} 7.25,7.62$ \\
\hline
\end{tabular}

\section{Peptide 3}

\begin{tabular}{|c|c|}
\hline Residue & NH \\
\hline \multicolumn{2}{|l|}{ Peptide 1} \\
\hline${ }^{\mathrm{a}}$ Ac-His $* 1$ & 8.52 \\
\hline Ala2 & 8.42 \\
\hline Ala3 & 8.27 \\
\hline His $* 4$ & 8.55 \\
\hline His $* 5$ & 8.78 \\
\hline Glu6 & 8.60 \\
\hline Leu7 & 8.44 \\
\hline His $* 8-\mathrm{NH}_{2}$ & 8.58 \\
\hline \multicolumn{2}{|l|}{ Peptide 2} \\
\hline${ }^{\mathrm{a}}$ Ac-His $* 1$ & 8.34 \\
\hline Ala2 & 8.22 \\
\hline Ala3 & 8.21 \\
\hline His*4 & 8.50 \\
\hline His $* 5$ & 8.63 \\
\hline Glu6 & 8.13 \\
\hline Leu7 & 8.15 \\
\hline His $* 8-\mathrm{NH}_{2}$ & 8.44 \\
\hline Peptide 3 & \\
\hline (T) & 8.7 \\
\hline
\end{tabular}

${ }^{\mathrm{a}}$ Ac-His*1

$8.79 \quad 4.26$

$3.13,3.21$

H2 7.80; H5 6.92; NMe 3.62;

${ }^{\mathrm{a}} \mathrm{CH}_{3} 2.14$

$\begin{array}{llll}\text { Ala2 } & 8.79 & 3.91 & 1.43 \\ \text { Ala3 } & 8.18 & 4.14 & 1.35\end{array}$




$\begin{array}{ccccl}\text { His*4 } & 8.23 & 4.41 & 3.21,3.24 & \mathrm{H} 27.62 ; \mathrm{H} 57.15 ; \mathrm{NMe} 3.43 \\ \text { His*5 } & 7.91 & 3.96 & 3.04,3.19 & \mathrm{H} 27.75 ; \mathrm{H} 56.23 ; \mathrm{NMe} 3.62 \\ \text { Glu6 } & 7.87 & 4.11 & 2.20 & \gamma \mathrm{CH}_{2} 2.56 \\ \text { Leu7 } & 7.72 & 4.14 & 1.78,1.82 & \gamma \mathrm{CH} 1.58 ; \delta \mathrm{CH}_{3} 0.98,1.08 \\ \text { His*8-NH} 2 & 7.21 & 4.30 & 3.26,2.73 & \mathrm{H} 27.69 ; \mathrm{H} 56.03 ; \mathrm{NMe} 3.65 ; \\ & & & & \mathrm{NH}_{2} 7.18,7.29\end{array}$

${ }^{\mathrm{a} A c}=\mathrm{acetyl}$

Table S2. ROE derived distance and ${ }^{3} J_{\mathrm{NHCH} \alpha}$ derived $\phi$-angle restraints used for calculating the solution structure of compound 3 in $90 \% \mathrm{H}_{2} 0: 10 \% \mathrm{D}_{2} 0$.

\begin{tabular}{|c|c|c|}
\hline Atom A & Atom B & Upper distance restraint $(\AA)$ and comment. \\
\hline $\mathrm{Ac}-\mathrm{CH}_{3}$ & His*1-NH & $4.2 ;$ Strong $+1.5 \AA$ correction \\
\hline His $* 1-\mathrm{H} \alpha$ & His*1-NH & 3.5; Medium \\
\hline His*1-H $\alpha$ & Ala2-NH & 3.5; Medium \\
\hline His* $1-\mathrm{H} \beta 1$ & His*1-NH & 3.5; Medium \\
\hline His $* 1-\mathrm{H} \beta 2$ & His*1-NH & 3.5; Medium \\
\hline His*1-H $\delta 1 \#$ & His*1-Hع 1 & 4.2; Strong + $1.5 \AA$ correction \\
\hline His*1-H $\alpha$ & Ala4-NH & 5.0; Weak \\
\hline His*1-H $\beta 1$ & His*1-H $\delta 2$ & 5.0; Weak \\
\hline His $* 1-H \beta 2$ & His $* 1-H \delta 2$ & 5.0; Weak \\
\hline His $* 1-\mathrm{H} \alpha$ & His*1-H $\delta 2$ & 2.7; Strong \\
\hline His $* 1-H \alpha$ & His $* 5-H \delta 2$ & 5.0; Weak \\
\hline His*1-Hع 1 & His*4-H $\delta 1 \#$ & 6.0 ; Weak + $1.5 \AA$ correction \\
\hline His*1-H $\delta 2$ & His*4-H $\delta 1 \#$ & 4.2; Strong $+1.5 \AA$ correction \\
\hline His*1-Hع 1 & His*5-Hع1 & $5.0 ;$ Weak \\
\hline His*1-H $\delta 2$ & His $* 5-H \delta 2$ & 5.0; Weak \\
\hline His*1-Nع2 & His $* 5-N \varepsilon 2$ & 2.9; As Pd coordination \\
\hline Ala2-H $\alpha$ & Ala2-NH & 3.5; Medium \\
\hline Ala2-H $\beta \#$ & Ala2-NH & 5.0; Medium $+1.5 \AA$ correction \\
\hline Ala2-H $\alpha$ & Ala3-NH & $5.0 ;$ Weak \\
\hline Ala2-H $\alpha$ & His $* 5-\mathrm{H} \delta 2$ & 3.5; Medium \\
\hline Ala2-H $\alpha$ & His $* 5-\mathrm{NH}$ & $5.0 ;$ Weak \\
\hline Ala2-H $\beta \#$ & Ala3-NH & 5.0; Medium $+1.5 \AA$ correction \\
\hline Ala2-NH & Ala3-NH & $5.0 ;$ Weak \\
\hline Ala3-H $\alpha$ & Ala3-NH & 2.7; Strong \\
\hline $\mathrm{Ala} 3-\mathrm{H} \alpha$ & Ala4-NH & $5.0 ;$ Weak \\
\hline Ala3-H $\beta \#$ & Ala3-NH & 4.2; Strong $+1.5 \AA$ correction \\
\hline Ala3-H $\beta \#$ & Ala4-NH & $5.0 ;$ Medium $+1.5 \AA$ correction \\
\hline Ala3-NH & Ala4-NH & $5.0 ;$ Weak \\
\hline $\mathrm{His}^{*} 4-\mathrm{H} \alpha$ & His*4-NH & 2.7; Strong \\
\hline $\mathrm{His} * 4-\mathrm{H} \alpha$ & His $* 5-\mathrm{NH}$ & 3.5; Medium \\
\hline His*4-Hß1 & His*4-NH & 2.7; Strong \\
\hline His $* 4-H \beta 2$ & His*4-NH & 2.7; Strong \\
\hline
\end{tabular}




\begin{tabular}{|c|c|}
\hline His*4-H $\beta \#$ & His $* 5-\mathrm{NH}$ \\
\hline His*4-Hס1\# & His $* 5-\mathrm{NH}$ \\
\hline His $* 4-\mathrm{H} \alpha$ & Leu7-HN \\
\hline His $* 4-\mathrm{H} \alpha$ & His $* 4-H \delta 2$ \\
\hline His $* 4-\mathrm{H} \alpha$ & His*8-H $\delta 2$ \\
\hline His*4-Hס1\# & His*4-Hع 1 \\
\hline His*4-H $\beta 1$ & His*4-H $\delta 2$ \\
\hline His*4-Hß2 & His*4-H $\delta 2$ \\
\hline His*4-Hס1\# & His $* 5-\mathrm{H} \delta 2$ \\
\hline His*4-H $\delta 2$ & Leu7-Hס\# \\
\hline His*4-NH & His*5-NH \\
\hline His*4-Hع1 & His*8-H $\delta 2$ \\
\hline His*4-Nع2 & His*8-Nع2 \\
\hline His $* 5-\mathrm{H} \alpha$ & His*5-NH \\
\hline His $* 5-\mathrm{H} \alpha$ & Glu6-NH \\
\hline His*5-H $\beta \#$ & His $* 5-\mathrm{NH}$ \\
\hline His*5-H $\beta \#$ & Glu6-NH \\
\hline His $* 5-\mathrm{H} \alpha$ & His $* 5-\mathrm{H} \delta 2$ \\
\hline His*5-H $11 \#$ & His $* 5-H \varepsilon 1$ \\
\hline His*5-H $\beta \#$ & His $* 5-\mathrm{H} \delta 2$ \\
\hline His*5-H $\delta 1 \#$ & His $* 5-H \delta 2$ \\
\hline Glu6-H $\alpha$ & Glu6-NH \\
\hline Glu6-H $\alpha$ & Leu7-NH \\
\hline Glu6-H $\beta \#$ & Glu6-NH \\
\hline Glu6-H $\gamma \#$ & Glu6-NH \\
\hline Glu6-Hß\# & Leu7-NH \\
\hline Glu6-NH & Leu7-NH \\
\hline Leu7-H $\alpha$ & Leu7-NH \\
\hline Leu7-H $\alpha$ & His*8-NH \\
\hline Leu7-H $\beta 1$ & His*8-H $\delta 2$ \\
\hline Leu7-H $\beta 2$ & His $* 8-H \delta 2$ \\
\hline Leu7-H $\gamma$ & Leu7-NH \\
\hline Leu7-H $\beta 1$ & Leu7-NH \\
\hline Leu7-H $\beta 2$ & Leu7-NH \\
\hline Leu7-NH & His*8-NH \\
\hline $\mathrm{His} * 8-\mathrm{H} \alpha$ & His*8-NH \\
\hline His $* 8-\mathrm{H} \alpha$ & His*8-H $\delta 2$ \\
\hline His*8-H $\delta 1 \#$ & His*8-Hع 1 \\
\hline His*8-Hß\# & His $* 8-H \delta 2$ \\
\hline His*8-Hß\# & His*8-NH \\
\hline His $* 8-\mathrm{H} \alpha$ & His*8-HT* \\
\hline
\end{tabular}

6.0; Weak + 1.0 A correction

6.0; Weak $+1.5 \AA$ correction

5.0; Weak

3.5; Medium

5.0; Weak

4.2; Strong + 1.5 A correction

5.0; Weak

5.0; Weak

4.2; Strong $+1.5 \AA$ correction

6.0; Medium $+1.5 \AA+1.5 \AA$ correction

5.0; Weak

5.0; Weak

2.9; As Pd coordination

3.5; Medium

5.0; Weak

3.3; Strong + $0.6 \AA$ correction

6.0 ; Weak $+1.0 \AA$ correction 5.0; Weak

4.2; Strong + 1.5 A correction

4.1; Medium + 0.6 A correction

6.0; Medium $+1.5 \AA$ correction

2.7; Strong

3.5; Medium

3.3; Strong $+0.6 \AA$ correction

5.6; Weak + $0.6 \AA$ correction

6.0; Weak + 1.0 A correction

5.0; Weak

3.5; Medium

5.0; Weak

6.0 ; Very weak

6.0 ; Very weak

5.0; Weak

5.0; Weak

5.0; Weak

5.0; Weak

5.0; Weak

5.0; Weak

4.2; Strong + $1.5 \AA$ correction

5.6; Weak $+0.6 \AA$ correction

5.6; Weak + $0.6 \AA$ correction

4.5; Medium + $1.0 \AA$ A correction

$\phi$-angle restraints

\begin{tabular}{ccc}
\hline Residue & ${ }^{3} J_{\mathrm{NHCH} \alpha}$ & $\phi$-restraint \\
\hline His*1 & $<2.0$ & $-65.0 \pm 25.0^{\circ}$ \\
Ala2 & $<2.0$ & $-65.0 \pm 25.0^{\circ}$ \\
Ala3 & 3.8 & $-65.0 \pm 25.0^{\circ}$
\end{tabular}




$\begin{array}{ccc}\text { His*4 } & 5.1 & -65.0 \pm 25.0^{\circ} \\ \text { His*5 } & 4.5 & -65.0 \pm 25.0^{\circ} \\ \text { Glu6 } & 4.5 & -65.0 \pm 25.0^{\circ} \\ \text { Leu7 } & 4.8 & -65.0 \pm 25.0^{\circ} \\ \text { His*8 } & - & -\end{array}$

\# Represents protons that were not stereospecifically assigned and whose distance restraints were adjusted with standard pseudoatom corrections (Wuthrich, K.; Billeter, M.; Braun, W. J. Mol. Biol. 1983, 169, 949). 